\title{
Fracturas del tercio superior facial. Experiencia en el Servicio de Cirugía de Cabeza, Cuello y Máxilo-Facial del Hospital Nacional "Dos de Mayo", 1999 - 2009
}

\author{
Superior third facial fractures at Dos de Mayo National Hospital Head, Neck and \\ Maxillo-Facial Surgery Service, 1999 - 2009
}

\begin{abstract}
Francisco Avello Canisto ${ }^{1,2}$, José Saavedra Leveau, ${ }^{1,2}$, Ladislao Pasache Juárez ${ }^{1}$, Roberto Iwaki Chávez', José Núñez Castañeda ${ }^{1}$, Marco Robles Hermenegildo ${ }^{1}$

Servicio de Cirugía de Cabeza, Cuello y Máxilo-Facial del Hospital Nacional Dos de Mayo.

${ }^{2}$ Departamento de Cirugia de la Universidad Nacional Mayor de San Marcos.
\end{abstract}

\begin{abstract}
Resumen
Introducción: Las fracturas del tercio superior facial corresponden a una patologia importante pero poco frecuente en la especialidad de cabeza, cuello y máxilo-facial, consecuencia de traumatismos severos; determinarlas por edad, sexo y agente causal ayudará para su manejo. Objetivos: Determinar la presentación de fracturas del tercio superior facial por edad, sexo, agente causal y clasificación. Diseño: Estudio observacional descriptivo. Lugar: Servicio de Cirugia de Cabeza, Cuello y Máxilo-Facial del Hospital Nacional Dos de Mayo, Lima-Perú. Participantes: Pacientes con fractura del tercio superior facial. Métodos: Recolección de datos registrados en las historias clínicas, entre junio 1999 y mayo 2009. Principales medidas de resultados: Edad, sexo, agente causal y clasificación. Resultados: Se encontró 49 pacientes, 90\% de sexo masculino, 67,5\% entre 21 y 40 años de edad, 55\% por accidentes de tránsito y $23 \%$ por robos; $41 \%$ eran solamente fracturas del tercio superior y $59 \%$ acompañadas del tercio medio facial. Conclusiones: Las fracturas del tercio superior facial son importantes en la región de la cara. El diagnóstico clínico depende de la estructura afectada. Se debe actuar oportunamente para evitar secuelas y complicaciones. Los accidentes de tránsito y robos son causas que van en aumento. Se espera que este trabajo ayude a elaborar protocolos de atención en servicios de emergencia.
\end{abstract}

Palabras clave: Tercio superior facial, región fronto-orbitaria, región frontal media, región fronto-parietal.

\section{Abstract}

Introduction: Superior third facial fractures represent an important but rare pathology in the specialty of head, neck and maxillofacial surgery, and result from severe trauma. Objectives: To determine the presentation of superior third facial fractures by age, sex, causal agent and classification. Design: Observational descriptive study. Setting: Head, Neck and Maxillofacial Surgery Service, Dos de Mayo National Hospital, Lima, Peru. Participants: Patients with superior third facial fracture. Methods: Medical records data between June 1999 and May 2009 were captured. Main outcome measures: Age, sex, causing agent and classification. Results: Forty nine patients were included. Ninety per cent were male, about two thirds (67.5\%) were between 21 and 40 years old. More than half of the lesions (55\%) resulted from traffic accidents and $23 \%$ from robberies; more than $40 \%$ had superior third fractures only and $59 \%$ were accompanied by midface fractures. Conclusions: Superior third facial fractures are important in the facial region. Clinical diagnosis depends on the affected structure. Treatment should be timely to prevent sequelae and complications. Traffic injuries and robberies are increasingly important causes. This study may contribute to developing treatment protocols in emergency services.

Keywords: Facial superior third, fronto-orbital region, middle frontal region, fronto-parietal region.

An Fac med. 2014;75(4):319-22 / doi: http://dx.doi.org/10.15381/anales.v75i4.10845

\section{INTRODUCCIÓN}

Cuando hablamos de tercio superior facial nos referimos prácticamente a toda la porción del hueso frontal que forma parte del esqueleto facial o de la cara (aproximadamente 2/3 partes del hueso frontal); es decir, excluimos la porción del hueso frontal que está por debajo del cuero cabelludo.

Las fracturas del tercio superior facial corresponden a una patología importante pero poco frecuente dentro de la especialidad de cirugía cabeza, cuello y máxilo-facial, como consecuencia de traumatismos severos en la región, los mismos que pueden ser de diversa etiología, comprometiendo especialmente el cerebro y el globo ocular por vecindad. La determinación de este tipo de fractura, por edad, sexo y agente causal, es importante para su manejo.

En la producción de este tipo de fracturas se requiere la acción de un traumatismo directo de mayor intensidad con un objeto contuso, generalmente consecuencia de accidentes de tránsito, agresiones por robo o durante peleas; las caídas en forma casual o por estado de ebriedad son importantes pero en menor frecuencia. Se debe tener en cuenta las heridas por proyectil de arma de fuego y explosivos, como indicador del aumento de la violencia en nuestro país. Afectan mayormente al sexo masculino y en especial entre los 20 y 40 años de edad ${ }^{(1,2)}$.

El tercio superior facial está conformado por el hueso frontal, el mismo que contiene a los senos paranasales frontales y contribuye a la formación de estructuras como la cavidad craneal, las órbitas y cavidad nasal. 
La clínica de estas fracturas dependerá de la porción ósea afectada. Presentan compromiso encefálico (traumatismo encéfalo-craneano) por la intensidad y vecindad del trauma. Igualmente se puede tener compromiso visual de intensidad variada, de acuerdo a la edad del paciente y sin olvidar las características locales inherentes a todo traumatismo: dolor, hematoma y edema facial. Muchas veces las fracturas no son aisladas o únicas, sino que son la combinación de varios elementos óseos fracturados. El diagnóstico confirmatorio es efectuado mediante estudios radiológicos por imágenes del cráneo, hueso frontal y órbitas, especialmente tomografía axial computarizada o helicoidal multicorte con reconstrucción tridimensional ${ }^{(2)}$.

Las secuelas y complicaciones de estas fracturas están condicionadas a diversas circunstancias, dentro de las que podemos distinguir especialmente las relacionadas con la intensidad del trauma y grado de compromiso cerebral y ocular, teniendo en cuenta la edad del paciente, la demora en el tratamiento, una mala colaboración del paciente (especialmente en los niños), una poco adecuada técnica quirúrgica empleada, una falla en la consolidación ósea, sobre todo en pacientes de edad avanzada, desnutridos, con alguna enfermedad sistémica concomitante o con un cuadro infeccioso local (osteomielitis, otros) ${ }^{(1,2)}$

Prácticamente, el hueso frontal posee tres porciones o regiones: región fronto-orbitaria, región frontal media y región fronto-parietal (figura 1). De esta forma podemos precisar con más claridad que el tercio superior facial comprende principalmente las regiones fronto-orbitaria y frontal media, respectivamente, equivalentes aproximadamente a $2 / 3$ partes del hueso frontal. La región fronto-orbitaria comprende los arcos superciliares y borde superior de las órbitas derecha e izquierda, es decir, va de lado a lado, incluyendo la prominencia frontal media o glabela y porción fronto-nasal; esta región forma el techo orbitario y encontramos en su espesor al seno frontal. La región fronto-parietal comprende la porción del hueso frontal que limita con los huesos parietales, siguiendo una distribución paralela a la unión fronto-parietal. La región frontal media comprende la porción que se encuentra entre las dos anteriormente descritas, incluye las eminencias frontales laterales derecha $\mathrm{e}$ izquierda. Por vecindad, en los traumas severos, puede haber compromiso del tercio medio facial y/o de otros huesos del cráneo. Estas fracturas pueden ser conminutas, con múltiples fragmentos, pudiendo comprometer más de una región. De acuerdo a lo mencionado, las fracturas del hueso frontal y del tercio superior facial pueden ser clasificadas de la siguiente forma ${ }^{(3)}$ (figura 1):

1. Fracturas de la región fronto-orbitaria:

1.a) Interna.

1.b) Externa.

2. Fracturas de la región frontal media:

2.a) Interna.

2.b) Externa.

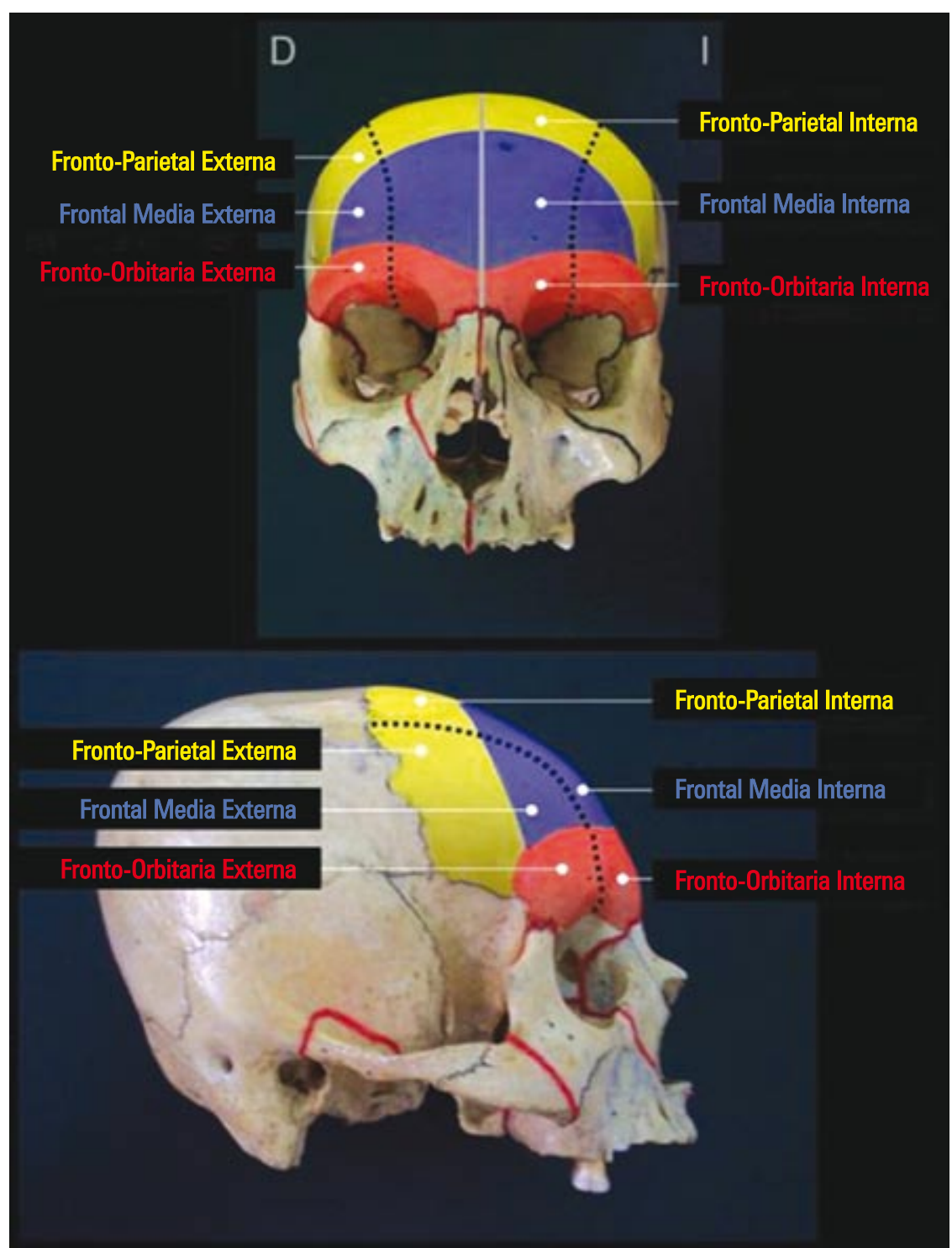

Figura 1. El hueso frontal forma el tercio superior facial. Véanse las zonas de fractura según la región afectada. Región fronto-orbitaria (color rojo), región frontal media (color azul) y región frontoparietal (color amarillo). Vista frontal y lateral. 
3. Fracturas de la región fronto-parietal:

3.a) Interna.
3.b) Externa.

Las fracturas del tercio superior facial son mayormente fronto-orbitarias (compromiso del seno frontal y del techo orbitario). En general, son de poca frecuencia en relación a otras fracturas faciales y pueden presentarse en forma aislada o acompañadas de fracturas del tercio medio.

\section{MÉTODOS}

Se revisó las historias clínicas de pacientes portadores de fractura del tercio superior facial, desde junio de 1999 hasta mayo de 2009, en el Servicio de Cirugía de Cabeza, Cuello y Máxilo-Facial del Hospital Nacional Dos de Mayo.

La recolección de datos se hizo mediante una ficha (previamente elaborada), con información necesaria para la investigación (fracturas del tercio superior, edad, sexo, agente causal y tipo de fractura), teniendo como finalidad determinar su frecuencia y clasificación.

El estudio realizado fue de tipo observacional descriptivo. Los datos obtenidos fueron revisados, clasificados y tabulados estadísticamente, en forma manual-electrónica, los mismos que son presentados en tablas (método de asociación), con la ayuda de programas de computación (Microsoft Word-Excel).

\section{RESULTADOS}

El total de historias clínicas revisadas de pacientes con 'fractura del tercio superior facial' fue 49 , equivalente a $5,4 \%$ de todas las fracturas faciales. Ocuparon el tercer lugar en frecuencia entre los tercios faciales, siendo el más frecuente el tercio medio. El 41\% correspondió solamente a fracturas del tercio superior, mientras que $59 \%$ se acompañaron de fracturas del tercio medio facial.

Tabla 1. Fracturas del tercio superior facial:frecuencia en porcentaje según edad y sexo (49 casos).

\begin{tabular}{|c|c|c|c|}
\hline \multirow{2}{*}{$\begin{array}{c}\text { Edad } \\
\text { (en años) }\end{array}$} & \multicolumn{2}{|c|}{ Sexo } & \multirow{2}{*}{$\begin{array}{c}\text { Tota } \\
(\%)\end{array}$} \\
\hline & Femenino (\%) & Masculino (\%) & \\
\hline 1 a 10 & 2 & 2 & 4 \\
\hline 11 a 20 & 0 & 4 & 4 \\
\hline 21 а 30 & 2 & 34,8 & 36,8 \\
\hline 31 a 40 & 4 & 26,7 & 30,7 \\
\hline 41 a 50 & 0 & 12,3 & 12,3 \\
\hline 51 a 60 & 0 & 8,2 & 8,2 \\
\hline 61 a 70 & 2 & 2 & 4 \\
\hline Total (\%) & 10 & 90 & 100 \\
\hline
\end{tabular}

Tabla 2. Fracturas del tercio superior facial: frecuencia en porcentaje según 'causa y sexo' (49 casos).

\begin{tabular}{cccc} 
& \multicolumn{2}{c}{ Sexo } & Total \\
Causa & Femenino $(\%)$ & Masculino (\%) & 55 \\
Acc. de tránsito & 6 & 49 & 23 \\
Robos & 2 & 21 & 12 \\
Casual & 0 & 12 & 10 \\
Peleas & 2 & 8 & 100 \\
\hline Total (\%) & 10 & 90 & 0 \\
\hline
\end{tabular}

Accidentes de tránsito: choque automovilístico y atropellos. Robos: objeto contuso y proyectil arma de fuego. Casual: caídas, agresión animal, proyectil arma de fuego.

Tabla 3. Fracturas del tercio superior facial: frecuencia en porcentaje según clasificación y sexo (49 casos).

\begin{tabular}{|c|c|c|c|c|c|}
\hline \multirow{2}{*}{ Clasificación } & \multicolumn{2}{c}{ Sexo } & \multicolumn{2}{c|}{$\begin{array}{c}\text { Total } \\
(\%)\end{array}$} \\
\cline { 2 - 5 } & Femenino (\%) & Masculino (\%) & \multirow{2}{*}{80} \\
\hline \multirow{2}{*}{$\begin{array}{c}\text { Región fronto- } \\
\text { orbitaria }\end{array}$} & Interna & 4 & 29 & 43 & \\
\cline { 2 - 5 } & Externa & 4 & 43 & 8 & \multirow{2}{*}{20} \\
\hline \multirow{2}{*}{$\begin{array}{c}\text { Región frontal } \\
\text { media }\end{array}$} & Interna & 2 & 6 & 12 & \\
\cline { 2 - 5 } & Externa & 0 & 12 & 900 \\
\hline \multicolumn{2}{|c|}{ Total (\%) } & 10 & 90 & \multicolumn{2}{|c|}{100} \\
\hline
\end{tabular}

El 90\% de los pacientes fue de sexo masculino y el $10 \%$ femenino. La edad de presentación fue desde los 5 hasta los 63 años; el 67,5\% estuvo entre los 21 y 40 años de edad (tabla 1).

El 55\% se debió a accidentes de tránsito (choque automovilístico y atrope1los), 23\% agresiones por robo (objeto contuso y proyectil arma de fuego), $12 \%$ casual (caídas, agresión animal, proyectil arma de fuego) y $10 \%$ peleas (tabla 2).

Las fracturas de la región frontoorbitaria fueron las más frecuentes
(80\%), mientras que las de la región frontal media obtuvieron 20\%. La fractura de mayor incidencia fue la frontoorbitaria externa con $47 \%$, seguida de la fractura fronto-orbitaria interna con $33 \%$ (tabla 3).

\section{DISCUSIÓN}

Las fracturas del tercio superior facial pueden ser de diversa etiología. Son las menos frecuentes de la región, pero su manejo es muy importante debido a su complejidad, por el grado de compro- 
miso cerebral y ocular con el que cursan, haciendo necesaria la evaluación conjuntamente con especialidades como neurocirugía y oftalmología. Es importante para su manejo una buena clasificación de las mismas; nos ayudará para un buen planeamiento de la cirugía y la aplicación de una técnica quirúrgica adecuada; ayudará también a elaborar protocolos de atención en los servicios de emergencia competentes, dependiendo directamente del tipo de fractura, la edad y el agente causal.

La gran mayoría de estas fracturas correspondió a fracturas de la región fronto-orbitaria, presentándose significativamente en forma combinada con el tercio medio facial. Tanto en las fracturas de la región fronto-orbitaria como en las de la región frontal media se afectó mayormente la porción externa. El sexo masculino fue el mayormente afectado, con notoria ventaja sobre el sexo femenino, siendo la edad de mayor incidencia entre los 21 y 40 años.

No se tuvo casos de fracturas de la región fronto-parietal, las mismas que coincidentemente por lo expuesto al inicio del trabajo excluimos del tercio superior facial y asumimos que han sido adecuada y oportunamente tratadas por la especialidad de Neurocirugía.

Los accidentes de tránsito son la principal causa de las fracturas del tercio superior facial; luego le siguen los robos, los mismos que pueden ser ocasionados por objeto contuso y por proyectil de arma de fuego; estas son causas que van en aumento como consecuencia de la violencia en la que actualmente vivimos, tanto delictiva como del tránsito vehicular.

El diagnóstico clínico depende de la estructura ósea afectada y se confirma mediante los estudios radiológicos pertinentes, ayudando también a la elección del material de osteosíntesis. En muchas ocasiones, el manejo de estos pacientes se debe hacer conjuntamente con neurocirugía y oftalmología, debido al compromiso encéfalo-craneano y óculo-orbitario, respectivamente, producto de la intensidad y de la localización del traumatismo.

Se debe actuar en forma oportuna para evitar secuelas y complicaciones, teniendo en cuenta la edad del paciente, demora en el tratamiento, no colaboración del paciente (especialmente en los niños) y a una falla en la consolidación ósea, sobre todo en pacientes de edad avanzada, desnutridos, con alguna enfermedad sistémica concomitante o infecciosa.

Debemos tener presente que contamos con un gran número de nuestra población que carece de recursos económicos o que es informal, con un problema serio de atención de salud por no contar con algún tipo de seguro; incluso en muchos de ellos las necesidades básicas están insatisfechas, con una estructura familiar deteriorada, y viven en condiciones de hacinamiento. Por lo general, son personas que están subempleadas o desempleadas, lo que nos limita principalmente en la disponibilidad de imágenes radiográficas adecuadas y en la adquisición del material necesario para cada tipo de fractura, con la consiguiente demora diagnóstica, retraso en el tratamiento y secuelas respectivas.

\section{REFERENCIAS BIBLIOGRÁFICAS}

1. Avello A. Cirugia de Cabeza, del Cuello y Maxilofacial. Lima, Perú: MAD Corp SA.; 2002.

2. Avello F. Epidemiologia y clasificación de las fracturas máxilo-faciales. Hosp. Nac. Dos de Mayo. Tesis de especialidad en Cirugia de Cabeza, Cuello y Máxilo-Facial. Lima, Perú: Universidad Nacional Mayor de San Marcos, Facultad de Medicina; 2002.

3. Avello F, Avello A. Nueva clasificación de las fracturas del tercio superior facial: consideraciones anátomo-quirúrgicas. UNMSM. An Fac Med. Lima. 2008; 69(4):272-7.

4. Espinosa JA. Generalidades de las fracturas faciales. Acta Otorrinolaring Cirug Cabeza Cuello. Bogotá, Colombia. 2003; 31(1):18-26.

5. Goñi I. Traumatismo de cara y cuello. Manual de Patologia Quirúrgica de Cabeza y Cuello. Pontificia Universidad Católica de Chile; 2004.
6. López-Arranz JS, Junquera LM, Villarreal P, Albertos JM. Urgencias maxilofaciales. Curso de formación continuada en medicina de urgencias. Hospital Central de Asturias. Oviedo, España.

7. Heredero S, Zubillaga I, Castrillo M, Sánchez G, Montalvo JJ. Tratamiento y complicaciones de las fracturas de seno frontal. Servicio de Cirugía Oral y Maxilofacial. Hospital Universitario 12 de Octubre. Madrid, España. Rev Esp Cirug Oral Maxilofac. 2007 mayo-junio; 29(3):145-53.

8. Sáinz de la Maza ML, Górriz G, González J Fracturas de tercio facial superior. Servicio de Cirugia Plástica. Hospital Na. Sra. de Aránzazu, San Sebastián y Hospital Central de Asturias, Oviedo. Manual on-line de Cirugia Plástica. Sociedad Española de Cirugia Plástica, Reparadora y Estética. Disponible en: www.secpre.org/documentos $\% 20$ manual\%2041.html.

9. Rougier J, Tessier P, Hervouety F, Woilez M, Lekieffre M, Derome P. Les fractures fronto-orbitaitres et du canal optique. Chirurgie Plastique Orbitopalpebrale. Masson. 1977:85-109.

10. Mason PN. Frontobasilar fractures en facial injuries. En: McCarthy JG. Plastic Surgery, volume 2. The face, Part 1. W.B. Saunders Co. 1990:1107-25.

11. Quetglas J, Rubio V. Fracturas del tercio superior de la cara. Traumatología Facial, Cirugia Plástica Ibero-Latinoamericana. Madrid. 1983:369-92.

12. Alberdi F. Índices pronósticos traumatológicos. Máster en Urgencias y Emergencias médicoquirúrgicas, Ed. Marco Garde P y col. Facultad de Medicina, Universidad del País Vasco. Madrid: Jarpyo Editores. 1990:152-9.

13. Madariaga N, Iglesias C, Legorburu MM, Gabilondo FJ. Fracturas faciales complejas. Manual de Cirugia Plástica. Sociedad Española de Cirugia Plástica, Reparadora y Estética. Disponible en: www.secpre.org/documentos $\% 20$ manual $\% 20$ 46.html.

14. Rouvière H. Compendio de Anatomia y Disección. 3ra. ed. reimp. Barcelona, España: Edit. Salvat; 1984.

Artículo recibido el 1 de abril de 2014 y aceptado para publicación el 1 de junio de 2014.

Conflicto de intereses:

Los autores niegan cualquier conflicto de interés.

Financiamiento propio.

\section{Correspondencia:}

Dr. Francisco Avello Canisto.

Servicio de Cirugia de Cabeza, Cuello y Máxilo-

Facial.

Hospital Nacional Dos de Mayo, Parque de la Medicina.

Av. Grau s/n (altura cuadra 13), Barrios Altos.

Lima 1, Perú.

Correo electrónico: favelloc@hotmail.com 